\title{
15. COMMISSION POUR L'ETUDE PHYSIQUE DES COMETES
}

\author{
Président: P. Swings.
}

Membres: MM. Baldet, Bertaud, Beyer, Biermann, Bobrovnikoff, Bouska, Dobrovolsky, Douglas, Dufay, Golay, Mme Herman, MM. Herzberg, Hunaerts, Levin, Lyttleton, McKellar, Merton, F. D. Miller, Minnaert, Oort, S. V. Orlov†, Poloskov, Richter, Rijves, Steavenson, van Biesbroeck, Vanysek, Vsekhsvyatsky, Waterfield, Whipple, Wurm.

\section{INTRODUCTION}

Le présent rapport n'a aucunement la prétention d'être complet: beaucoup trop de travaux ont été publiés sur la physique cométaire depuis I954 pour qu'on puisse les mentionner tous. Nous nous baserons essentiellement sur les notes reçues des membres de la Commission, en essayant surtout d'attirer l'attention sur quelques concepts nouveaux introduits récemment, comme les rôles possibles des radicaux libres.

La dernière période triennale a été caractérisée par l'apparition de plusieurs comètes brillantes, notamment Arend-Roland (I956h) et Mrkos (I957 d) ; ces comètes spectaculaires ont suscité un intérêt considérable dans le grand public. Dans les milieux astronomiques, la physique cométaire a pris une place de choix, illustrée par les colloques qui lui ont été consacrés, les nouveaux livres et périodiques publiés, les instruments installés pour l'observation des comètes.

Dans les colloques de Liège de I954, 'Les particules solides dans les astres', et r956, 'Les molécules dans les astres', une place importante a été réservée aux comètes. Il en a été de même au colloque international sur la matière interplanétaire tenu à Jena et Weimar en octobre r957, mais dont nous ne possédons pas encore les comptes rendus.

En U.R.S.S. un Bulletin de la Commission pour l'étude des comètes et météores est publié par le Conseil Astronomique de l'Académie des Sciences; le premier numéro est paru en I957.

Le livre Statistik und Physik der Kometen de N. Richter a été traduit en anglais par Beer et Lyttleton, après avoir été misà jour par l'auteur et les traducteurs. Vsekhsvyatsky a terminé et publié son catalogue complet des magnitudes absolues [r]; on y trouve les $\mathrm{H}_{10}$ (parfois $\mathrm{H}_{0}$ et $n$ ) de toutes les comètes du Catalogue de Baldet, plus ceux des nouvelles comètes jusqu'en I956. Ce catalogue qui a déjà été utilisé par divers chercheurs constitue une partie d'une monographie de Vsekhsvyatsky Caractéristiques Physiques des Comètes qui paraîtra en fin 1957 et où sont décrites toutes les principales observations cométaires. Baldet a tenu à jour son Catalogue général des orbites et sa Liste des comètes; il a écrit le livre v, 'Comètes, Météores et Météorites', de l'Astronomie Populaire (I955, pp. 331-404), ainsi que la section sur les comètes dans l'Encyclopédie française, t. III (I956).

A plusieursobservatoires, la recherche de comètes nouvelles et la redécouverte de comètes périodiques constitue une des activités importantes; Mrkos a remporté un succès particulier (découverte de I955e, I956 $b$, I957 $d$; redécouverte de r955i et I956 $a$ ). Le nombre d'amateurs intéressés aux observations cométaires s'est aussi considérablement accru.

Beaucoup de nouveaux instruments ont été installés: les chambres pour la photométrie monochromatique de N. Richter, la camera Baldet d'objectif $f / \mathrm{I}$ de $\mathrm{I} 7 \mathrm{~cm}$, le prisme objectif à défilement de film de Madame R. Herman, les prismes-objectifs de l'Observatoire de Haute Provence (O.H.P.), le spectrophotomètre direct de Liller, etc....

\section{NOTE SUR LA RECOMMANDATION ADOPTEE PAR L'ASSEMBLÉE GÉNERALE À ROME}

Cette recommandation était la suivante:

La Commission attribue une grande importance à la publication d'un atlas photographique de spectres cométaires typiques. Elle exprime sa gratitude à $\mathrm{Mr}$ Swings qui veut bien s'occuper de la réalisation d'un tel atlas. Elle sollicite de l'U.A.I. un subside, etc.... 


\section{ETUDE PHYSIQUE DES COMETES}

Déjà à la réunion de Zurich (I948) la publication d'un tel Atlas avait été discutée, mais aucune résolution n'avait été prise.

Le travail de préparation commencé dès l'automne I952 a été terminé en fin I955. L'Atlas of Representative Cometary Spectra a été envoyé en I956 aux membres de la Commission I5 et à la plupart des observatoires. Les auteurs sont P. Swings et L. Haser. L'ensemble des planches est précédé d'un exposé général sur les spectres cométaires et d'une description des bandes essentielles. Le travail a été effectué grâce à un subside de l'A.R.D.C. (U.S. Air Force).

\section{DIMENSIONS, ASPECT}

Les publications d'ordre descriptif ont été extrêmement nombreuses. Ce fut le cas notamment pour les comètes brillantes I956h et $1957 d$ qui ont fait l'objet de nombreux articles, certains d'ensemble, dans maintes revues (Sky and Telescope, Observatory, Astronomie, Hemel en Dampkring, etc...). On a observé des aiguillons (queues dans la direction du soleil) à maintes comètes (voir rapport pour Dublin); mais aucun n'a jamais été aussi spectaculaire que celui de la comète I956 $h$. L'explication de cet aiguillon a, sans doute, été imaginée par maints observateurs; elle a été publiée, entre autres, par F. L. Whipple [2] et par G. Larsson-Leander [3]. Nous ne nous attarderons pas ici aux descriptions détaillées de $1956 h$ et $1957 d$, d'autant plus que beaucoup de travaux à leur sujet sont à l'impression ou en préparation. De sérieux progrès ont été faits dans la photographie: on a utilisé des filtres colorés, parfois même des filtres interférentiels; on a calibré les photographies en vue de la photométrie. Grâce à l'emploi de filtres, on a pu, par exemple, séparer nettement les queues à $\mathrm{CO}^{+}$et à $\mathrm{Na}$, de la queue de poussière, de $\mathrm{I} 957 d[4]$. On a mesuré les mouvements dans la queue de $1956 h$ [s].

La distribution de la luminosité dans la tête a été étudiée théoriquement par $\mathrm{D}$. $\mathrm{O}$. Mocknatsch [6], L. V. Wallace [7] et L. Haser [8]. Des mesures photo-électriques de Th. Widorn (Vienne) ont fourni, pour de nombreuses observations de $1956 \mathrm{~h}$, les magnitudes correspondant à 4 diaphragmes allant de $0 !^{\prime} 5$ à $I^{\prime} 5$ et ont permis à J. Hopmann de trouver une loi de décroissance $R^{-\frac{1}{4}}$ de la brillance dans la tête. L'aspect ovale de la coma de la comète Honda (I955g) a été mis en évidence et suivi par Waterfield jusqu'au moment de la séparation annoncée du noyau en deux parties (Miss Roemer).

Les observations les plus développées sont celles de F. D. Miller qui observe toutes les comètes dont l'image au télescope Curtis Schmidt est assez grande pour être analysée à l'isophotomètre; depuis I955, ce fut le cas de I955 $e$, I955g, I956h, I957 d et I957c (Encke). Depuis 1957, Miller a ajouté à ses filtres en verre et en gélatine, un filtre interférentiel isolant la bande $\lambda_{5} \mathrm{r}_{5} \mathrm{de}_{2}$. Il va y ajouter un filtre correspondant au continuum solaire et un autre isolant $\mathrm{CO}^{+}$. Tous les clichés sont calibrés; une comparaison a, d'ailleurs, été faite avec une série de sections faites dans $1955 \mathrm{~g}$ avec le photomètre photoélectrique de Liller. Par voie photoélectrique, Schmidt et Van Woerden (Leiden) déterminent les intensités monochromatiques dans des cercles concentriques, et effectuent des sections radiales donnant la distribution monochromatique d'intensité dans la tête. On commence aussi à effectuer des mesures monochromatiques dans la queue. Van Woerden a observé photo-électriquement I953g, I954 $f$, I955e, I955 $f$, I955g, I956b, I956a, I956h, I957d et I957c (Encke).

J. Bouška a étudié la distribution de la luminosité dans la tête de la comète r955e. Il s'occupe de déterminer les isophotes dans la tête de r $956 h$.

V. P. Konopleva (Publ. Obs. Astr. Kiev, vol. XI) a étudié la distribution de brillance et évalué la distribution de densité dans la tête de plusieurs comètes.

Beyer (Bergedorf) a continué ses observations de structure de queue et ses mesures de diamètre de coma. A. M. Bakharev (Stalinabad) a déterminé les types de queue, selon Bredichine, pour plusieurs comètes, d'après leur forme et leur disposition [ro]. G. $K$. Nazartschouk [n] a réexaminé les observations de nuages au sein des queues et, notamment, les mouvements de ces nuages dans I943I : il obtient des accélérations considérables. Par contre, S. V. Orlov [12] trouve qu'il n'y a ni des accélérations gigantesques, ni de 


\section{COMMISSION 15}

grandes vitesses initiales. Orlov, réexaminant le problème des queues de type $\mathrm{I}\left[\mathrm{CO}^{+}\right]$ conclut que la pression de radiation est responsable pour leur formation.

W. G. Rijves ( $P u b l$. Obs. Astr. Tartu, vol. 33, no. 2, à l'impression) a complété son étude des accélérations répulsives des queues de type I. En partant des mouvements de formations nuageuses, il trouve pour la force répulsive du soleil des valeurs de I50-300 (Morehouse), 40-Ioo (Swift et Halley).

Un point de vue très différent est défendu dans le groupe de L. Biermann. $P$. Stumpff [13] a montré que la combinaison stéréoscopique de photographies de queue révèle, non pas le mouvement relatif de la comète et de la terre, mais bien le changement de structure de la queue entre les deux expositions. En fait, l'effet stéréoscopique permet de déterminer les déplacements au sein de la queue, avec plus de précision que les mesures séparées des clichés. Stumpff a déterminé les mouvements des gaz de la queue de la Comète Morehouse le 29 Octobre I908. Les régions proches de l'axe semblent moins accélérées par radiation solaire corpusculaire que les parties extérieures où des accélerations allant jusque $\mathrm{ro}^{4}$ sont trouvées.

H. Alfvén [14] pense que les lignes de force magnétiques jouent un rôle important dans les comètes. La radiation corpusculaire solaire consisterait en faisceaux avec champ magnétique gelé, comme on considère dans certaines théories des aurores. Les hautes accélérations observées seraient dues à des forces électromagnétiques.

\section{PHOTOMÉTRIE}

Durant ces dernières années, le photomètre photo-électrique, d'habitude associé à des filtres, a été de plus en plus utilisé, conformément au vœu exprimé à Dublin et à Rome. Il en est souvent résulté un accroissement de précision et de sensibilité. D'ailleurs, en photométrie photographique aussi bien que photo-électrique, l'emploi de filtres isolant des bandes déterminées ou le rayonnement solaire a permis de donner un sens physique plus précis aux observations. Nous n'en sommes pourtant encore qu'au début dans ce domaine.

Parmi les installations photo-électriques actives en photométrie cométaire, citons celles de Leiden (Schmidt et Van Woerden), Bergedorf (Thiessen), Pic du Midi, Haute Provence, Vienne, Brno. Même des amateurs avertis ont dirigé leurs observations dans cette voie (exemple: J. P. Legrand, à Colombes, France). Beaucoup de comètes récentes ont été observées photo-électriquement, mais ces observations n'ont, en général, pas encore été discutées soigneusement. Une discussion détaillée a été faite par Thiessen pour ses observations photo-électriques de I956h. En particulier, Thiessen trouve que la tête de I956h est plus rouge que le Soleil; étant donné toutefois que Thiessen a utilisé des filtres larges, on ne peut guère corriger ses mesures d'indice de couleur de l'effet des bandes moléculaires. Des observations photo-électriques de la comète I957c (Encke) ont été effectuées à l'Observatoire de Haute Provence par Mianes; il sera extrêmement intéressant de les reprendre à de prochains retours de la comète.

Une instrumentation photographique très complète a été installée par N. Richter. Plusieurs chambres à court foyer ont été équipées de filtres interférentiels à bandes passantes de roo $\AA$, centrées à $\lambda \lambda 6532,6270,6050,5889,5553,5165,4737,4380,4088$, 3883. On peut, d'ailleurs, aussi placer des filtres polarisants. Les instruments permettent d'atteindre la magnitude 8. Pour $1956 h, 260$ expositions furent obtenues au cours de 27 nuits!

Beyer a continué efficacement ses mesures visuelles de magnitude de nombreuses comètes (I 8 comètes depuis I953c). Merton exprime son impression que les meilleures estimations visuelles sont effectuées avec des instruments dont l'ouverture est juste suffisante pour montrer les comètes clairement. Baldet a continué à développer sa méthode extrafocale. Ch. Bertaud a fait de nombreuses et excellentes mesures photographiques pour I955 $g$, I956b, I956h. H. Grenat, J. Fraleux et R. Herman [15] ont déterminé la courbe de lumière de $1956 h$ à partir de spectres pris au prisme objectif, comparés à des étoiles du champ ayant un type spectral voisin de celui du Soleil. 


\section{ETUDE PHYSIQUE DES COMETES}

Les mesures photographiques de magnitude et d'indice de couleur sont continuées à Vienne par A. Purgathofer.

Pour la photométrie du noyau, les méthodes visuelles avec un très fort grossissement restent encore requises; de telles mesures ont été effectuées, notamment pour I956h, par J. Hopmann au réfracteur de $68 \mathrm{~cm}$ de Vienne, avec un grossissement 260 fois. La loi

$$
I=I_{0} \cdot f(\alpha) \cdot \Delta^{-2} \cdot r^{-2}
$$

où $\alpha$ est l'angle de phase, a donné $f(\alpha)=$ constante pour $\alpha$ variant de 28 à I2 $4^{\circ}$ dans I956h. Hopmann remarque qu'il y a un intervalle de ro dans les magnitudes absolues des noyaux.

On sait qu'il y a souvent désaccord entre les magnitudes visuelles déterminées avec un petit instrument et les magnitudes photographiques déterminées avec un gros instrument lorsque la comète est devenue plus faible.

Les déterminations de magnitude absolue et de coefficient $n$ ont continué à faire l'objet des préoccupations de Vsekhsvyatsky, Vodopianova, Vanyšek, Bouška, Beyer. Parmi les magnitudes absolues les plus élevées, on trouve $1955 b\left(\mathrm{H}_{10}=4 \cdot 8\right)$; parmi les plus faibles, I $953 e$ et ${ }^{2} 954 j\left(\mathrm{H}_{10}=\mathrm{I} 4 \cdot 8\right)$. Vanyšek et $\mathrm{A}$. Hruška ont discuté à nouveau la variation du coefficient $n$ et l'ont rapprochée du pourcentage variable de poussière dans la tête.

\section{POLARISATION}

Pour la première fois, le président de la Commission $\mathrm{I}_{5}$ a la satisfaction de signaler de nombreuses observations de polarisation, effectuées par diverses techniques et avec des récepteurs variés (photo-électriques ou photographiques). On attendait impatiemment de telles mesures qui étaient notamment désirables pour comprendre l'origine du spectre continu. Les observations concernent toutes $1956 h$ et $1957 d$. Peu sont complètement réduites et publiées.

A Cambridge (Angleterre) D. E. Blackwell et R. V. Willstrop [16] ont procédé photographiquement en isolant le continuum (près de $\lambda 4530$ ) et la bande o-o de $C N$ $(\lambda 3850)$. Ils ont couvert un intervalle d'angle de phase de $26^{\circ}$ et trouvé une polarisation de 5 à $20 \%$ (comète $\mathrm{I} 956 \mathrm{~h}$ ). La courbe de polarisation dans le continuum ressemble à celle d'un météorite métallique; le pourcentage de polarisation dans $\mathrm{CN}$ est d'accord avec une excitation fluorescente. Les observations ont été faites au foyer principal du télescope de 36 pouces, en dédoublant l'image au moyen d'un gros prisme de calcite.

Madame Martel a observé I957d toutes les nuits du I8 au 29 août au moyen d'un polarigraphe du type d'Öhman monté au foyer Cassegrain du réflecteur de $80 \mathrm{~cm}$ de 1'O.H.P. Les observations comptent II2 plages photographiées dans 9 domaines spectraux; il s'agit de la tête et de son voisinage immédiat. La polarisation de la queue est supérieure à celle de la tête; elle semble être la même dans toutes les longueurs d'onde. Par contre, la direction du plan de polarisation varie rapidement d'un bord à l'autre sur l'éventail de la queue. Les résultats quantitatifs ne sont pas encore obtenus; pour $\mathrm{Na}$ et le proche infra-rouge, la polarisation est de l'ordre de $20 \%$.

Madame Elvius a aussi utilisé un polarigraphe d'Öhman pour observer I956h et I957d à l'astrographe Zeiss de $40 \mathrm{~cm}$ de Stockholm; aucun filtre n'était interposé (émulsion I03 $\mathrm{aO}$ ). La polarisation était positive (vecteur électrique le plus grand perpendiculaire au plan soleil-comète-terre). Pour I957 $d$, le degré de polarisation est environ $25 \%$. Pour I $956 \mathrm{~h}$, la queue donne I8 à $20 \%$, de même que la partie de la tête dirigée à l'opposé du soleil; il semble que la partie de la tête dirigée vers le soleil ait une polarisation moins grande, ro à $15 \%$, mais ceci est incertain.

N. Richter a couvert une très vaste variation de l'angle de phase $\alpha$, de 1 i 6 à $36^{\circ}$ pour I956h (du 22 avril au 3 juin). La polarisation de la tête varie fortement avec $\alpha$; le pourcentage va jusque $45 \%$. Des mesures furent aussi faites par Gütler à Munich.

M. K. Vainu Bappu et S. D. Sinvhal (Observatoire d'Uttar Pradesh, Naini Tal, Indes) [r7] ont observé photo-électriquement r $956 h$ et r $957 d$, en utilisant des filtres colorés standards et des filtres interférentiels centrés à $\lambda \lambda 5890,5000,4700$ et 4300 (demi bande passante: 


\section{COMMISSION 15}

$80 \AA$ ); une lame de polaroid pouvait occuper six positions. De nombreuses et excellentes valeurs du pourcentage de polarisation et de la position du plan de vibration ont été trouvées. Les résultats sont en accord avec ceux qu'a obtenus Ohman en I94r.

M. Blaha et Z. Svestka (I956h), et A. Hruška et V, Vanyšek (I957d) ont aussi fait des déterminations de polarisation. Il en est de même de Th. Walraven et M. de Vries (Leiden, mesures photo-électriques de I956h), de Th. Widorn (Vienne, mesures photoélectriques de $1956 h$ du 25 avril au 23 mai; maximum de polarisation de $22 \%$ pour $\alpha=90^{\circ}$ ) et de Rozhkovski (Alma-Ata).

\section{SPECTROSCOPIE}

L'apparition des deux comètes brillantes en 1957 est venue bien à point pour fournir des spectres à fente permettant de faire progresser les identifications.

Certes, les spectres à fente de $1955 e$, I $955 f$ et $1955 g$ pris à Victoria par McKellar présentaient de l'intérêt; ces trois comètes avaient des émissions de $\mathrm{CN}, \mathrm{C}_{3}, \mathrm{C}_{2}$ et $\mathrm{CH}$ assez fortes et un faible continuum solaire. Il n'empêche qu'aucun progrès substantiel n'avait été effectué dans les identifications cométaires au cours des dernières années. Avant l'apparition de I956h, B. Rosen, P. Swings et L. Houziaux [r8] avaient procédé à une discussion systématique de toutes les molécules dont la présence dans les comètes possédait quelque caractère de vraisemblance. Aucune conclusion nette n'avait pu être tirée, principalement parce que les longueurs d'onde des émissions cométaires sont trop peu précises, particulièrement dans la région visible et le proche infra-rouge. $\mathrm{P}$. Swings et $L$. Haser [זg] avaient également examiné, sans résultat positif, si la raie brillante $L \alpha$ du soleil peut exciter une fluorescence de molécules cométaires.

On pouvait espérer qu'un effort serait fait pour obtenir des spectres à fente de $1956 h$ et $1957 d$. Certes, ces deux comètes dont les spectres se ressemblent fort, n'étaient pas idéales pour la détection et la mesure d'émissions faibles. Toutes deux présentaient un fort continuum solaire, dont l'intensité totale était beaucoup (au moins trois fois) supérieure à l'intensité totale des bandes. Soit dit en passant, toutes deux avaient aussi une émission du sodium qui était anormalement intense et, au moins pour I956h, extrêmement variable; sur plusieurs spectrogrammes (Palomar, Michigan,...) la structure double de l'émission D était nettement visible. Pour les deux comètes, la région visible fut bien couverte à Victoria et à l'O.H.P. A. McKellar a mesuré une cinquantaine d'émissions de $\lambda 5000$ à $\lambda 6700$ dans 1957 $d$ et constaté leur accord avec l'identification antérieure de $\mathrm{NH}_{2}$. Ch. Fehrenbach, $\mathrm{L}$. Haser, P. Swings et A. Woszczyk [20] ont mesuré un grand nombre de spectrogrammes de $1956 h$ pris avec un réseau par transmission et ont obtenu, dans la région visible, des longueurs d'onde plus précises que celles qui étaient connues jusqu'ici. Ils ont ainsi pu discuter de façon plus approfondie, l'identification de $\mathrm{NH}_{2}$, grâce surtout à l'excellente analyse de laboratoire faite par D. A. Ramsay. Pour I957d , P. Swings, J. Dufay et L. Haser [22] ont obtenu plusieurs spectrogrammes s'étendant jusque $\lambda 8900$ et montrant une structure triple des émissions voisines de $\lambda 7900$ et $\lambda 8$ Ioo découvertes dans $1947 n$ et I947 $k$. Cette structure a permis d'attribuer les émissions cométaires de façon certaine aux transitions $(2-0)$ et $(3-I)$ du système rouge de $\mathrm{CN}$. Les clichés de I957d pris à l'O.H.P. permettront d'autres recherches spectrophotométriques.

Aussi bien pour I956h que pour I957 $d$, la bande (o-o) $\lambda 3880$ de $\mathrm{CN}$ révélait une structure complexe sur les clichés de Victoria et de l'O.H.P. C'est, toutefois, sur les spectrogrammes obtenus par J. L. Greenstein au 200-inch du Palomar que la structure rotationnelle de la bande (o-o) de $\mathrm{CN}$ apparaît avec un détail extraordinaire grâce à la haute dispersion ( $\mathrm{I} 8 \AA / \mathrm{mm}$ ) et l'excellente définition du spectrographe. Cette structure sera étudiée théoriquement par J. Hunaerts. Les clichés de Greenstein s'étendent jusque dans le rouge; leur dispersion de $27 \AA / \mathrm{mm}$ dans la région de $\mathrm{NH}_{2}$ permet d'obtenir des longueurs d'onde beaucoup plus précises que toutes celles dont on a disposé jusqu'ici. La structure des bandes de $C_{2}$ pourra être étudiée. Bref, ces documents spectroscopiques 


\section{ETUDE PHYSIQUE DES COMETES}

feront sûrement progresser de façon appréciable notre connaissance de la physique cométaire. Sans doute, pourra-t-on en discuter à la réunion de la Commission is à Moscou.

Une belle série de spectres de la comète r957c (Encke) a aussi été obtenue à l'O.H.P. par P. Swings, Ch. Fehrenbach et A. Woszczyk [22]. Comme en I957, la comète d'Encke n'avait pratiquement pas de spectre continu (faisant ainsi contraste avec r $956 h$ et $1957 d$ ), alors que les émissions moléculaires, notamment celle de $C_{3}$, étaient intenses. Une comparaison a été effectuée entre les spectres de I937, I947 et I957.

Il est certain que des spectres à fente de $1956 h$ et $1957 d$ ont dû être obtenus en de nombreux observatoires. Parmi les spectres dont nous possédons quelque description, citons ceux de I956h pris par Madame Herman à l'O.H.P. [15] (spectrographe à prisme attaché au réflecteur de $120 \mathrm{~cm}$, étude de l'intensité relative des bandes par rapport au fonds continu); ceux de I956h et I957 $d$ obtenus à Ann Arbor par T. P. Stecher et D. B. McLaughlin (région visible avec plus haute dispersion et région ultraviolette); ceux de I956h pris par W. P. Bidelman à l'Observatoire Lick (notamment région visible avec $90 \AA / \mathrm{mm}$, excellente définition des raies les plus intenses de $\mathrm{NH}_{2}$ ); ceux de $\mathrm{G}$. H. Herbig au Lick (réflecteur Crossley, spectres de la tête et du début des queues), ceux de C. Y. Fan à l'Observatoire McDonald ( 9 au spectrographe B et 3 au Coudé, 2 de ces derniers allant de 3700 à $5000 \AA$ avec une dispersion de $36 \AA / \mathrm{mm}$, le 3ème de 4500 à $7000 \AA$ avec une dispersion de $72 \AA / \mathrm{mm}$; il semblait que la raie D apparaissait, dans la tête, uniquement du côté opposé au soleil). Aumoment de la rédaction du rapport, tout ce matériel était à l'étude.

I956h et I957 $d$ offraient d'intéressants problèmes à traiter au prisme-objectif, particulièrement $\mathrm{r} 957 d$ dont les queues de gaz $\left(\mathrm{CO}^{+}\right)$(filaments ondulés à peu près rectilignes et condensations) et de poussière (queue large, diffuse, fortement courbée) pouvaient ainsi être complètement différenciées, de même que l'émission du sodium (filaments minces et droits). Les observations correspondantes de $1957 d$ ont été effectuées notamment à l'O.H.P. par J. H. Bigay, N. H. Doan et M. Dufay [4] (en combinaison avec des photos avec filtres colorés à des télescopes Schmidt); à Meudon par F. Baldet [23] et par Madame Herman; à Stalinabad par O. V. Dobrovolsky et ses collaborateurs; en Crimée par Vsekhsvyatsky et ses collaborateurs. Des observations d'autres comètes (notamment I $956 h$ et I957c) ont été effectuées au prisme-objectif par J. Dufay et Mlle M. Bloch (Lyon), Ch. Bertaud (Meudon), Madame R. Herman [15] (Meudon, dont un P. O. avec défilement de film), Z. Ceplecha, J. Rajchl, J. Bouška et K. Hermann-Otavský [24] (Ondřejov), M. Beyer [25] (Bergedorf), S. Vsekhsvyatsky et ses collaborateurs (Crimée), Parijsky (Alma Ata), F. D. Miller [26] (Michigan). Nous avons appris que de nombreux autres observatoires d'U.R.S.S. ont effectué des observations au prisme-objectif, mais nous ne possédons pas de détail à ce sujet.

Il est, peut-être, intéressant de signaler les renseignements que nous avons reçus au sujet du spectre de la queue et de l'aiguillon de $1956 \mathrm{~h}$. La queue avait un continuum très intense auquel étaient superposées les émissions habituelles, relativement faibles, de

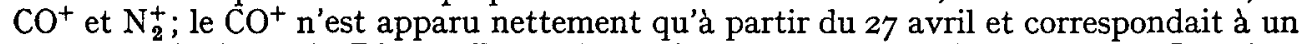
rayon très fin (Beyer). D'après Beyer, le continuum correspondait à une étoile $\mathrm{G}_{3}$, alors que d'après Liller (voir plus loin), il était plutôt du type $\mathrm{K} 5 \mathrm{~V}$. Il semble bien que le caractère général du spectre de l'aiguillon (continuum intense, bandes faibles) soit identique à celui de la queue. Stecher place le maximum diffus du spectre de l'aiguillon aux environs de $\lambda 4700$. Des observations spectroscopiques de la queue et de l'aiguillon ont aussi été effectuées par G. H. Herbig à l'Observatoire Lick.

Un effort est fait à plusieurs observatoires pour l'enregistrement photo-électrique direct du spectre des comètes. A notre connaissance, le plus grand succès, jusqu'à présent, a été remporté par W. Liller qui a surtout étudié $1956 h$, I957d et I957c (Encke) au télescope Curtis de l'Université de Michigan. Pour I956h, Liller a, au cours de plusieurs nuits, enregistré la tête de $\lambda 3400$ à $\lambda 6400$, puis jusque $\lambda$ I2 000; il a aussi enregistré la queue à différentes distances du noyau, de $\lambda 3400$ à $\lambda 6400$ et l'aiguillon dans le même domaine spectral. La queue a essentiellement un spectre continu dont la couleur est celle d'une 


\section{COMMISSION 15}

étoile $\mathrm{K}_{5} \mathrm{~V}$. Si ce continuum est dû à la diffusion par des particules sphériques, le diamètre moyen de celles-ci doit tomber entre 0.5 et $4 \mu$ (dépendant de leur indice de réfraction). Liller estime aussi l'abondance de ces poussières, qui est requise pour expliquer la brillance de la queue.

Pour I $957 d$, Liller a effectué des observations semblables, mais moins nombreuses. Le spectre de la tête de I957d est presque identique à celui de $1956 h$. Des tracés s'étendant jusque $\lambda$ I2 ooo révèlent une émission voisine de $\mathrm{I} \mu$; il s'agit, sans doute, de la bande $(0-0)$ du système rouge de $\mathrm{CN}$.

Les observations de la comète d'Encke montrent que le continuum est extrêmement faible, en accord avec les spectrogrammes obtenus à l'O.H.P.

La comparaison des spectres théoriques et des spectres cométaires a continué à faire l'objet de travaux de J. Hunaerts qui a traité le cas de $\mathrm{NH}$ [32] par une méthode analogue à celle qu'il avait suivie pour $\mathrm{OH}$ et $\mathrm{CH}$. Les résultats sont semblables. Hunaerts s'occupe à présent de l'analyse de la structure de la bande (0-0) de CN dans I957d (clichés de J. L. Greenstein).

L'observation radio-spectroscopique des comètes a, enfin, conduit à un résultat positif dans le cas de $1956 h$ au voisinage de son périhélie. Il semble bien que le fond continu de la radio-émission cométaire ait été observé sous forme de 'bursts' par J. D. Kraus [27] (Ohio) sur $27.6 \mathrm{Mc} / \mathrm{s}$ et par H. G. Müller, W. Priester et G. Fischer (Bonn) sur I420 Mc/s. Au paraboloïde de $7.50 \mathrm{~m}$ de Humain (Belgique), une émission à caractère stable a été obtenue sur $600 \mathrm{Mc} / \mathrm{s}$ par A. Koeckelenbergh [28]; celle-ci a été attribuée par R. Coutrez, $\mathrm{J}$. Hunaerts et A. Koeckelenbergh [29] à un passage entre les sous niveaux dus au dédoublement $\Lambda$ du niveau $J^{\prime \prime}=1 \cdot 5$ de l'état ${ }^{2} \Pi_{3} / 2$ de la molécule $\mathrm{CH}$. Le caractère monochromatique de l'émission observée à Humain expliquerait l'absence d'emission à $400 \mathrm{Mc} / \mathrm{s}$ trouvée au réflecteur de 25 mètres de Dwingeloo (Hollande); il en est de même pour les observations négatives de Cambridge. On sait que les travaux de S. M. Poloskov [30] indiquent qu'on pourra difficilement détecter une radiation thermique continue; qu'une radiation non thermique (effet de plasma) est moins improbable; mais que la meilleure possibilité est offerte par les radiations monochromatiques résultant de transitions entre les composantes de structure fine dues au dédoublement $\Lambda$ des niveaux rotationnels. Les molécules $\mathrm{CH}$ et $\mathrm{OH}$ sont les plus aptes à être détectées. Pour $\mathrm{CH}$, la longueur d'onde calculée par Coutrez, Hunaerts et Koeckelenbergh, à partir des spectres optiques est de $50 \cdot 0 \mathrm{~cm}$, au lieu de $45.45 \mathrm{~cm}$ donnée par Poloskov.

O. V. Dobrovolsky [31] a récemment montré que, en tenant compte des oscillations de plasma excitées par l'interaction du flux corpusculaire solaire avec l'atmosphère cométaire, on peut s'attendre à une radio-émission de ces queues.

\section{TRAVAUX DE LABORATOIRE SUR DES MOLÉCULES COMÉTAIRES}

Certains spectres intéressants des comètes sont décrits dans le rapport de la sous-commission $29 b$ (Bandes moléculaires dans les spectres stellaires). Les principaux autres travaux venus à notre connaissance ont été effectués à Ottawa (G. Herzberg); Washington (Nat. Bureau of Standards, groupe de H. P. Broida), Université de Western Ontario (groupe de R. W. Nicholls) et Meudon (Mme R. Herman).

Le travail spectroscopique le plus intéressant pour les comètes est l'analyse détaillée du spectre de $\mathrm{NH}_{2}$ effectuée par D. A. Ramsay [33]. Les autres spectres de radicaux libres étudiés à Ottawa sont ceux de $\mathrm{HCO}_{\text {[34] }}$ et $\mathrm{HNO}$ [35]. Le spectre d'absorption de $\mathrm{CH}_{3}$ a été trouvé à $\lambda 2163$; son caractère diffus permet de comprendre que $\mathrm{CH}_{3}$, une fois formé, se décompose rapidement en $\mathrm{CH}_{2}+\mathrm{H}$. Il semble aussi que $\mathrm{CH}_{2}$ présente une absorption continue, de sorte que sa photo-décomposition conduirait à la formation du radical $\mathrm{CH}$ abondant dans les comètes [36]. Dans la photolyse par flash du diacétylène $\mathrm{HC} \equiv \mathrm{C}-\mathrm{C}$ $\equiv \mathrm{CH}$, le spectre du radical $\mathrm{C}_{3}$ a été observé en absorption. Quoiqu'on ne puisse pas en conclure que le diacétylène est la molécule parente du $\mathrm{C}_{3}$ cométaire, on peut, en tout cas, déclarer que $\mathrm{C}_{3}$ peut être formé par photo-décomposition d'hydrocarbones [37].

L'étude des radicaux libres constitue l'activité primordiale d'un nouveau groupe du 


\section{ETUDE PHYSIQUE DES COMETES}

Bureau of Standards (H. P. Broida), de même que d'autres laboratoires, notamment de G. C. Pimentel (Berkeley), de R. G. W. Norrish (Cambridge), de G. Porter (Sheffield) etc.... On verra, plus loin, l'intérêt croissant apporté au rôle possible des radicaux libres dans les comètes.

Dans la collection de travaux sur les phénomènes de combustion d'intérêt astrophysique publiée par l'Université de Western Ontario, G. V. Marr [38] a récemment fait paraître une étude des flammes oxyacétyléniques du point de vue de l'émission du radical $\mathrm{C}_{3}$. Marr compare le comportement des radicaux $\mathrm{C}_{3}$ dans les différentes sources de laboratoire, soit que celles-ci fournissent un continuum en plus du groupe 4050 (flammes, four de King), soit qu'elles donnent seulement l'émission discrète 4050 (décharges). Il examine l'émission $\mathrm{C}_{3}$ cométaire (discrète) et les absorptions (discrète et continue) dans les étoiles $\mathrm{N}$, en les comparant aux sources de laboratoire. Il couvre un vaste domaine spectral et ne trouve aucune émission de $C_{3}$ dans la région $5900-6800$.

A Meudon, Madame Herman a repris des spectrogrammes du groupe 4050 à grande dispersion et compte les étudier. Ses essais dans le rouge n'ont, jusqu'à présent, pas donné de résultat.

\section{QUELQUES CONSIDÉRATIONS SUR LE NOYAU DES COMETES ET LEUR ÉVOLUTION}

Dans son article de I949 B. J. Levin [39] a déjà supposé que des gaz gelés sont présents dans les noyaux des comètes.* Mais c'est $\mathrm{F}$. L. Whipple qui a montré le premier tout le parti qu'on pouvait tirer d'un modèle d' 'icy conglomerate'. Ce modèle a généralement reçu un accueil satisfaisant, quoique certains chercheurs, notamment $R$. A. Lyttleton, continuent à y présenter des objections. En fait, le problème de la constitution des noyaux cométaires est loin d'être complètement éclairci.

On parle généralement de formation d'une atmosphère cométaire par évaporation (sublimation) moléculaire. Levin [40] attire l'attention sur le fait qu'on ne doit pas croire qu'il existe une véritable couche de glaces à la surface du noyau. Selon lui, la croûte extérieure serait essentiellement composée de pierres ou poussières subsistant des couches glacées précédemment vaporisées. Il ne s'agirait donc pas d'une simple sublimation, mais plutôt d'une désorption de gaz contenus dans la couche superficielle. Pour les comètes à courte période, comme la comète d'Encke, la régénération gazeuse dans les couches extérieures se ferait par l'apport de gaz produits dans les couches glacées internes lorsque celles-ci sont atteintes (avec un retard assez long) par le rayonnement solaire. Ces vues de Levin ne sont pas universellement admises; d'autres ont été exposées par H. C. Urey et B. Donn [4 I].

On sait que certaines comètes subissent des augmentations d'éclat subites et considérables; par exemple I925 II (Schwassmann-Wachmann) a des éruptions de 2 à 5 magnitudes, deux ou trois fois par an. N. Richter pense qu'il existe une relation entre ces éruptions cométaires et l'activité solaire. C. Whitney [42] considère que, même si un bombardement solaire suscite l'éruption cométaire, celle-ci doit s'expliquer par une cause interne. A la distance héliocentrique 5.5 U.A. (cas de I925 II) la température d'équilibre est $130^{\circ} \mathrm{K}$; la seule molécule parente active est $\mathrm{CH}_{4}$ qui doit s'évaporer de la surface. La croûte extérieure contiendrait des pierres et des glaces inactives. Cette croûte est surement peu résistante. La création d'une concentration accidentelle de $\mathrm{CH}_{4}$ peut donner lieu à une poche à forte pression et conduire à une explosion qui expulserait une partie de la crô̂te extérieure et mettrait à nu des glaces actives. Pour une comète plus proche du soleil, d'autres molécules, comme $\mathrm{CO}_{2}$, peuvent devenir actives au même titre que $\mathrm{CH}_{4}$.

Ce mécanisme de Whitney présuppose des glaces pures de $\mathrm{CH}_{4}, \mathrm{CO}_{2}$, etc...; la situation différerait si on faisait appel à des hydrates solides. D'ailleurs, il faudrait que

* La présence de glaces dans les noyaux cométaires a été suggérée par Vsekhsvyatsky en 1948 (A. Zh. 25, 256, 1948). 


\section{COMMISSION 15}

la texture du noyau soit très résistante pour que puisse se constituer une poche de gaz à haute pression. B. Donn et $\mathrm{H}$. C. Urey $[43,4 \mathrm{I}]$ ont plutôt pensé à des explosions chimiques dans lesquelles les radicaux libres jouent le rôle essentiel. De nombreux travaux expérimentaux récents ont montré qu'on peut emprisonner des radicaux libres à basse température et que leur réchauffement peut provoquer des réactions en chaîne explosives. Il est, d'ailleurs, possible qu'un flux de protons solaires 'allume' une telle explosion.

L. Haser [44] attire l'attention sur le rôle que pourraient jouer des radicaux libres comme $\mathrm{OH}, \mathrm{CN}, \ldots$, emprisonnés dans les cristaux de $\mathrm{H}_{2} \mathrm{O}, \mathrm{CH}_{4}, \mathrm{NH}_{3}, \ldots$ ou dans les hydrates solides. Lorsque la comète se rapproche du soleil, il y aurait, non seulement sublimation (ou désorption) de $\mathrm{H}_{2} \mathrm{O}, \mathrm{CH}_{4}$, etc...., mais encore des radicaux les plus volatils. On est tenté de penser que des radicaux $\mathrm{CN}$ ou $\mathrm{OH}$ observés à grande distance héliocentrique sont dégagés directement comme radicaux. Des réactions chimiques pourraient, d'ailleurs, se produire entre radicaux; ceux-ci pourraient allumer des espèces de 'flammes' à basse pression. Il n'est pas impossible que le radical $\mathrm{C}_{3}$ existe à l'état emprisonné dans le noyau [33].

Quelque soit la structure physique du noyau, on conçoit bien qu'une diminution séculaire d'éclat et une désintégration finale puissent résulter de la formation continue d'une atmosphère dissipante. Les travaux dans cette voie se sont continués. Dobrovolsky [45] trouve que la magnitude de Pons-Brooks diminue d'une magnitude par révolution (I8II-I884-I953). Vsekhsvyatsky [46] conclut statistiquement que les comètes ont une désintégration rapide. V. I. Cherednichenko a examiné la question de la vitesse d'évaporation des glaces à la distance de Jupiter et conclu à l'impossibilité de l'existence de telles glaces. A. Hruška [47] conclut aussi que la vie d'une comète à courte période (5 ans), ayant un noyau d'un kilomètre de diamètre est de l'ordre de $10^{4}-10^{5}$ années. Dans son ouvrage Théorie physique des météores et de la matière météorique dans le système solaire (r956), B. J. Levin discute la formation des courants météoriques à partir de la désintégration des noyaux cométaires.

Il ne semble pas y avoir de doute que les comètes à courte période doivent avoir une vie courte. Ceci ne signifie évidemment pas que toutes les comètes sont nées récemment.

Lorsqu'on détermine la diminution séculaire de magnitude, on doit, comme l'a montré Dobrovolsky [48], en partant du Catalogue de Vsekhsvyatsky, être très prudent et tenir compte des conditions de visibilité de la comète périodique et, notamment, de sa hauteur moyenne au-dessus de l'horizon de l'observateur.

\section{I'ORIGINE DES COMETTES}

Etant donné qu'une réunion de la Commission I 5 à Moscou sera consacrée à l'origine des comètes, il ne paraît pas opportun de s'étendre ici sur les travaux à ce sujet. Il reste toujours, en présence, les trois types principaux d'hypothèse qui supposent, soit que les comètes sont apparues à l'époque de la formation des planètes, soit qu'elles ont été creées lors de processus secondaires ultérieurs, soit que leur production se continue encore actuellement.

Au colloque de Jena, B. J. Levin a défendu l'hypothèse d'une constitution du nuage de comètes de Oort lors de la formation du système solaire, dans la ligne de la théorie cosmogonique développée par $\mathrm{O}$. J. Schmidt. Au contraire, J. Witkowski y a parlé de la capture des comètes de l'espace interstellaire [49]; il ne partage pas les vues de Oort.

J. G. Tyror [50] a effectué une étude de la distribution des périhélies de 448 comètes à longue période. Il semble que les périhélies se groupent de préférence vers le plan galactique. Les résultats de Tyror sont en accord avec une formation des comètes par accrétion de poussière interstellaire (Lyttleton, Bondi).

Vsekhsvyatsky a continué à défendre sa théorie de formation éruptive des comètes [sr]. Celle-ci a été critiquée par B. J. Levin au Colloque de Jena, ainsi que par d'autres astronomes soviétiques dans d'autres circonstances. 


\section{ETUDE PHYSIQUE DES COMETES}

K. A. Steins $(A . Z h .34, \mathbf{1}, 86$, I957) a considéré les critères de capture par Jupiter et Saturne et apporté de nouveaux arguments en faveur de l'origine des comètes à courte période comme résultat de capture.

Ces questions pourront être discutées en détail à Moscou.

\section{RELATIONS AVEC L'ACTIVITÉ SOLAIRE}

L'Année Géophysique Internationale a attiré une attention spéciale sur la possibilité d'utiliser les comètes comme corps d'épreuves du champ solaire. La question est pourtant encore bien vague et incertaine. Deux effets possibles des rayonnements solaires sont à envisager essentiellement: une modification de la structure des queues (et, peut-être, des têtes) et une variation de la brillance de la tête.

L'idée de Biermann (accélération des ions dans les queues à $\mathrm{CO}^{+}$due à l'interaction entre le rayonnement corpusculaire solaire et le plasma de la queue), développée par divers astronomes a déjà été exposée dans le rapport pour le Congrès de Dublin. De nouveaux travaux théoriques sont venus s'y ajouter $\left[52, \mathrm{I}_{4}\right]$. Dobrovolsky [53] a également montré que la dispersion élastique des protons solaires dans les atmosphères cométaires peut provoquer une augmentation de température et l'apparition d'un halo.

Donn et Urey [4] ont montré, en se basant sur leurs idées relatives au rôle des radicaux libres, que l'excitation d'éruptions lumineuses du genre de celles de I925 II peut être due aux particules solaires. Biermann [54] est même arrivé récemment à l'hypothèse de l'identité complète du gaz interplanétaire et du rayonnement corpusculaire solaire; dans ce dernier, la densité en particules peut être estimée par le taux de développement des structures ioniques dans les queues cométaires.

V. I. Cherednichenko a étudié l'influence des émissions corpusculaires solaires sur les conditions physiques et la brillance des comètes.

Quant aux observations, elles sont extrêmement nombreuses, mais jamais tout-à-fait convaincantes. Bakharev [55] a constaté une augmentation brusque de brillance de $0.8 \mathrm{mag}$. de I955 $f$, qui correspond à une période d'activité solaire. La comète $1956 c$ a été retrouvée à la position calculée, mais beaucoup trop brillante [56]. Les astronomes de l'Observatoire Yerkes ont observé dans la queue de $1957 d$, des taches lumineuses, irrégulières et variables qui sont, peut-être, causées par des jets de particules solaires [57]. S. Orlov [58] a repris les éruptions lumineuses du 'noyau' de la comète Halley observées par Campbell et pense que celles-ci sont dues à des jets de particules solaires. Dans la comète Morehouse, Dobrovolsky [59] a déterminé statistiquement les vitesses des groupes de nuages de la queue gazeuse. Le 2 octobre I908, les accélérations étaient différentes des autres jours et l'activité géomagnétique était, de loin, supérieure. Les seules mesures photométriques précises, discutées en relation avec l'activité solaire, sont celles que G. Thiessen [60] a faites de la comète $1956 h$, par voie photo-électrique avec deux écrans colorés durant la période 23 avril à 30 mai I957. Thiessen trouve une corrélation entre la brillance cométaire et les nombres relatifs de taches, en ce sens qu'un accroissement du nombre des taches provoquerait une réduction de brillance. Thiessen suppose que cette réduction est due à une augmentation du rayonnement ultraviolet causant une plus forte photodissociation ou photo-ionisation. Les observations de Thiessen sont d'excellente qualité; elles sont malheureusement très peu nombreuses ( 7 sur 38 jours).

L. Houziaux et L. Battiau [6r] ont essayé de voir s'il existait une corrélation entre les 'flares' solaires et les variations de luminosité de cinq comètes récentes. Aucune corrélation n'a été trouvée. Les auteurs concluent qu'il est impossible de prouver l'existence d'une corrélation aussi longtemps qu'on ne possède pas de longue série d'observations monochromatiques précises de brillance de comètes.

Nous nous associons à cette conclusion; étant donné l'augmentation du nombre de photomètres photo-électriques consacrés aux comètes, il y a tout lieu de penser qu'une solution convenable sera bientôt donnée au problème de la corrélation entre la brillance des comètes et les phénomènes solaires. 


\title{
COMMISSION 15
}

SUGGESTIONS

Un grand nombre de suggestions émises à Dublin ou même à Rome et à Zurich restent valides. Reprenons celles de Rome:

(I) subsiste, mais commence à être réalisée;

(2) l'émission $\lambda_{7906}$ est identifiée (2-o du système rouge de $\mathrm{CN}$ ), mais la région $\lambda_{3600}$ reste à étudier;

(3) subsiste intégralement;

(4) subsiste partiellement; depuis Dublin, $\mathrm{NH}_{2}$ et $\mathrm{CH}_{3}$ ont été étudiés;

(5) subsiste;

(6) (7) et (8) ont fait quelque progrès;

(9) devrait être discutée à Moscou;

(I0) à (I4) subsistent, quoique quelques travaux aient été effectués.

Quant aux suggestions de Dublin:

(I) et (3) subsistent; pour le (3), un gros effort est fait;

(2) a été appliquée à des comètes récentes;

(4) a été réalisée;

(5) devrait être discutée à Moscou;

(6) cette discussion aura lieu à Moscou.

Les suggestions qui suivent précisent certains de ces points et en soulèvent quelques autres.

I. Du point de vue spectroscopique, un effort devrait être fait pour obtenir les spectres des queues dans l'ultra-violet, le visible et le proche infra-rouge; la région ultra-violette des têtes, voisine de $\mathrm{CN}$ ( $\mathrm{I}-0$ ) devrait être couverte avec une plus haute résolution. Quelques clichés à la haute dispersion du Palomar permettraient de résoudre maints problèmes en suspens dans tous les domaines spectraux.

2. Du point de vue photométrique, on a grandement besoin de longues séries de magnitudes monochromatiques précises qu'on pourra essayer de mettre en relation avec les phénomènes solaires.

3. N. Richter et L. Houziaux insistent sur la nécessité de continuer de bonnes mesures de polarisation, avec filtres adéquats, afin d'arriver à déterminer la dimension et la nature des particules donnant lieu à la lumière continue; le rôle joué par la diffusion par les électrons libres devrait aussi être discuté plus complètement.

4. Vsekhsvyatsky préconise la discussion des problèmes d'observation et d'investigation en collaboration dans le domaine de l'astronomie cométaire, de l'étude des météores, de la géologie et de la vulcanologie. Nous suggérons qu'une telle discussion ait lieu à Moscou lors de la séance sur l'origine des comètes.

5. Vsekhsvyatsky rappelle que les problèmes cométaires sont étroitement liés à des questions importantes concernant le soleil et l'ionosphère étudiées durant l'A.G.I. Il suggère de renouveler la proposition du Congrès de I938 à Stockholm (organisation de services d'observation physique des comètes). Un premier pas dans cette voie serait l'édition d'un Bulletin International pour la publication urgente des observations et des résultats préliminaires sur les particularités physiques des comètes. Nous pouvons rapprocher cette proposition de celle faite par Waterfield à Dublin.

6. Vsekhsvyatsky propose de rassembler sous la direction de la Commission 15 et de publier sous forme d'ouvrage, les photos, les spectres et, si possible, les déterminations photométriques se rapportant aux comètes $1956 h$ et I957 $d$. Il suggère qu'un subside soit, dans ce but, demandé à l'Union.

7. Il est souhaitable que les travaux expérimentaux sur les radicaux cométaires soient continués activement.

\author{
P. SWINGS \\ Président de la Commission
}




\section{ETUDE PHYSIQUE DES COMETES}

\section{RE F ÉRENCES}

[I] Vsekhsvyatsky, S. $A . Z h .33$, fasc. 4, 516, 1956.

[2] Whipple, F. L. Nature, 179, 1240, 1957.

[3] Larsson-Leander, G. Observatory, 77, 132, 1957.

[4] Bigay, J. H., Doan, N. H. et Dufay, M. C.R. Acad. Sci. Paris, 245, 921 , 1957.

[5] Wenske, K. Communication privée de M. Beyer. Miller, F. D. Communication privée.

[6] Mocknatsch, D. O. Bull. Inst. d'Astroph. Theorique, U.R.S.S., 6, no. 5, 269, 1956, et références $\mathrm{y}$ indiquées.

[7] Wallace, L. V. Astr. J. 6r, I9I, 1956.

[8] Haser, L. Bull. Acad. Belg. 43, 740, 1957.

[9] Miller, F. D. P.A.S.P. 69, 82, r957.

[10] Bakharev, A. M. Stalinabad Obs. Bull. no. 19, 1957, et références y indiquées.

[II] Nazartschouk, G. K. Communication privée de Vsekhsvyatsky.

[12] Orlov, S. V. A. Zh. 34, 2, 1957, et communication privée.

[13] Stumpff, P. A.N. 283, 193, 1956.

[14] Alfvén, H. Tellus, 9, 92, 1957.

[15] Grenat, H., Fraleux, J. et Herman, R. C.R. Acad. Sci. Paris, 245, 785, 1957.

[16] Blackwell, D. E. et Willstrop, R. V. M.N.R.A.S. I17, 590, 1957.

[17] Vainu Bappu, M. K. et Sinvhal, S. D. Nature, r8o, I4Io, I 957.

[18] Rosen, B., Swings, P. et Houziaux, L. Ann. Astrophys. 20, 76, 1957.

[19] Swings, P. et Haser, L. Ann. Astrophys. 20, 52, 1957.

[20] Fehrenbach, Ch., Haser, L., Swings, P. et Woszczyk, A. Ann. Astrophys. 20, I45, I957.

[21] Swings, P., Dufay, J. et Haser, L. C.R. Acad. Sci. Paris, 245, 1765, 1957; Swings, P. et Dufay, J. C.R. Acad. Sci. Paris, 246, I I21, 1958; Ann. Astrophys. 21, 26o, $195^{8}$.

[22] Swings, P., Fehrenbach, Ch. et Woszczyk, A. C.R. Acad. Sci. Paris, 245, 21 52, 1957.

[23] Baldet, F. C.R. Acad. Sci. Paris, 245, 923, 1957.

[24] Bouška, J., et Hermann-Otavský, K. Bull. Astr. Insts. Czech. vol. 9, no. I, I957.

[25] Beyer, M. A.N. 282, 145, 1955.

[26] Miller, F. D. Astr. J. 60, 173, 1955; P.A.S.P. 67, 402, 1955; 69, 82, 1957; Yoss, K. M. P.A.S.P. 67, 407, 1955 .

[27] Kraus, J. D. Circulaive U.A.I. no. I596, 29 avril 1957.

[28] Koeckelenbergh, A. Circulaire U.A.I. no. I594, I I avril 1957.

[29] Coutrez, R., Hunaerts, J. et Koeckelenbergh, A. Proc. Inst. Rad. Engrs, N.Y., à l'impression.

[30] Poloskov, S. M. A. Zh. 20, 1, 68, 1953; Coll. Liège, 1956, p. I12; voir aussi rapport Dublin.

[3I] Dobrovolsky, O. V. Stalinabad Obs. Bull. no. 23, 1957.

[32] Hunaerts, J. Coll. Liege, r956, p. 82.

[33] Ramsay, D. A. J. Chem. Phys. 25, 188, 1956; Coll. Liege, 1956, p. 47 I.

[34] Herzberg, G. et Ramsay, D. A. Proc. Roy. Soc. A, 233, 34, 1955.

[35] Dalby, F. W. non publié.

[36] Herzberg, G. et Shoosmith, J. Canad. J. Phys. 34, 523, 1956; Herzberg, G. Coll. Liege, I956, p. 397.

[37] Callomon, J. H. et Ramsay, D. A. Canad. J. Phys. 35, I29, I957.

[38] Marr, G. V. Combustion Studies of Astrophysical Significance, no. III, Ier mars 1957.

[39] Levin, B. J. Prirada, no. Io, 3, 1949.

[40] Levin, B. J. Rapport au Colloque International de Jena-Weimar sur la matière interplanétaire, octobre, 1957.

[4I] Donn, B. et Urey, H. C. Coll. Liege, 1956, p. 124.

[42] Whitney, C. $A p . J .122,190,1955$.

[43] Donn, B. et Urey, H. C. $A p . J .123,339,1956$.

[44] Haser, L. C.R. Acad. Sci. Paris, 241, 742, 1955; Bull. Acad. Belg. 42, 813, 1956.

[45] Dobrovolsky, O. V. Stalinabad Obs. Bull. no. I3, I955.

[46] Vsekhsvyatsky, S. Bull. Comm. Comètes et Metéorites, no. I, 5, I957. 


\section{COMMISSION 15}

[47] Hruška, A. Bull. Astr. Insts. Czech. 8, 10, I957.

[48] Dobrovolsky, O. V. Stalinabad Obs. Bull. no. 19, 1957.

[49] Pour un exposé plus ancien de Witkowski, J. sur cette question, voir Bull. Soc. Amis Sciences et Lettres de Poznañ, Série B, livraison XII, 1953.

[50] Tyror, J. G. M.N.R.A.S. 1r7, 370, 1957.

[5I] Vsekhsvyatsky, S. A. Zh. 34, fasc. 4, I957.

[52] Dobrovolsky, O. V. Stalinabad Obs. Bull. no. 18, 1956; et référence [31].

[53] Dobrovolsky, O. V. A. Zh. 32, 5I4, 1955.

[54] Biermann, L. Observatory, 77, I09, 1957.

[55] Bakharev, A. M. Astr. Circ. U.S.S.R. no. 178, 12 mars 1957.

[56] Harvard Announcement Card no. ${ }^{3} 34$.

[57] Sky and Telescope, 17, 27, 1957.

[58] Orlov, S. V. A. Zh. 33, 377, I956.

[59] Dobrovolsky, O. V. Stalinabad Obs. Bull. no. 18, 1956.

[6o] Thiessen, G. Z. Ap. 43, 260, I957; 44, I69, I958.

[6r] Houziaux, L. et Battiau, L. Bull. Acad. Belg. 43, I71, 1957.

\section{Compte rendu des Séances. I4 et I9 août r958}

PrÉSIDENT: P. Swings.

SECRETAIRE: K. Wurm.

Première Séance. I4 août

Le Président passe le Draft Report brièvement en revue, en essayant de le mettre à jour. Il considère surtout les suggestions; outre les remarques indiquées dans le rapport, il faut mentionner les points suivants.

\section{A. SUGGESTIONS ÉMISES À ROME}

(4) On souhaite spécialement connaître le spectre de $\mathrm{C}_{2}^{+}$.

(5) Il serait même intéressant de procéder, simplement, à des déterminations de couleur et de polarisation pour la comète Schwassmann-Wachmann.

(8) A propos de la brillance des noyaux, Hopmann fait rapport sur les observations visuelles faites à Vienne, ainsi que sur sa discussion de la question (voir Publ. Obs. Wien, I958); les magnitudes absolues des noyaux varieraient entre 5 et 20 .

(9 à I4) Les photos suggérées ont commencé à être obtenues. La proposition d'envoi d'informations physiques sur les comètes doit être rapprochée de la proposition de Veskhsvyatsky discutée ci-dessous (point $C_{5}$ ), ainsi d'ailleurs que de la proposition faite par Waterfield à Dublin.

\section{B. SUGGESTIONS ÉMISES À DUBIIN}

Plusieurs membres avaient insisté sur l'intérêt des mesures de polarisation; un excellent départ a été pris dans cette voie à l'occasion de l'apparition de $1956 h$ et $1957 d$.

(I) Bon début de réalisation.

(2) Bon début.

(3) Divers laboratoires s'occupent activement des radicaux gelés (notamment au U.S. Bureau of Standards). Quant à la détermination des sections efficaces des molécules cométaires pour l'excitation par chocs d'électrons ou de particules lourdes, elle commence à être étudiée théoriquement (notamment par Cherednichenko) et expérimentalement (en partie à cause de son intérêt pour les aurores polaires).

(5) Suggestion de Rijves de rassembler les reproductions de photos de comètes ayant des queues bien développées, se trouvant dispersées dans de nombreux observatoires (aucune décision prise à Dublin, en l'absence de Dr Rijves). 


\section{ETUDE PHYSIQUE DES COMETES}

La discussion de cette question indique que la Commission I 5 appuie cette suggestion. Il est proposé que le Dr Rijves se charge lui-même de la tâche. A la séance du Ig août, Dr Rijves exprime son accord et reçoit les remerciements du Président.

Il est entendu que la collection devrait être éventuellement mise à la disposition d'autres chercheurs, par exemple par envoi de copies.

\section{NOUVELLES SUGGESTIONS INDIQUÉES DANS LE RAPPORT}

(I, 2 et 3 ) Accord unanime.

(5) Vsekhsvyatsky expose ses vues qui recueillent l'assentiment des membres présents, notamment de J. Dufay qui insiste pour que soient indiquées les heures des photos, afin qu'on puisse suivre les formations sur des photos prises à des longitudes différentes.

Après discussion des différentes façons possibles de faire paraître un Bulletin International pour la publication rapide des observations physiques, il est proposé que Vsekhsvyatsky se charge de cette publication et qu'il prenne tous arrangements nécessaires à cet effet. Vsekhsvyatsky accepte cette mission et reçoit les remerciements du président. En réponse à une question de Richter, sur les langues dans lesquelles le Bulletin serait publié, il est suggéré par Vsekhsvyatsky que le Bulletin pourrait paraître en anglais, français, russe et allemand.

(6) Il est proposé que Vsekhsvyatsky étudie les possibilités de préparer la monographie suggérée et fasse, au Congrès de Ig6I, une proposition relative au subside à demander pour la publication éventuelle. Le travail sera considérable; le commission remercie Vsekhsvyatsky qui veut bien s'en charger.

Le Draft Report est alors adopté à l'unanimité.

Commencent ensuite de courts exposés sur des travaux récents.

F. L. Whipple rappelle le caractère de fragilité et de très faible densité $\left(\sim 0 \cdot \mathrm{I} \mathrm{g} / \mathrm{cm}^{3}\right)$ des météorites issus des comètes. Il montre que des courants météoritiques cométaires différents ont des caractéristiques différentes, sans doute en relation avec le caractère du noyau cométaire produisant le courant.

A propos de la communication de Whipple, P. M. Millman signale la présence de $\mathrm{H} \alpha$ dans de nombreux spectres météoriques (ex.: perséides); $\mathrm{H} \alpha$ n'est probablement pas produit par l'hydrogène atmosphérique. D'autres remarques sont faites par Levin, Richter, Wurm, Biermann et Poloskov.

\section{Deuxième Séance. I9 août}

Biermann expose des travaux effectués récemment par Stumpff sur les structures des queues; cet exposé suscite des remarques de Wurm.

Bouigue expose les mesures photoélectriques de I956h qu'il a faites à Toulouse; il compare la tête avant et après passage au périhélie; il décrit la distribution de lumière dans la tête, notamment en ce qui concerne le $\mathrm{C}_{2}$.

Dobrovolsky indique que les fluctuations de la brillance cométaire observées par Beyer sont en meilleure corrélation avec la phase du cycle undécennal du soleil qu'avec les nombres relatifs de taches. Suivent des remarques de Wurm, Richter et Vanyšek.

Vsekhsvyatsky expose ses vues sur la formation de synchrones dans la queue de la comète I957d; les bandes observées dans la queue courbe ne sont pas dirigées vers le noyau, mais bien vers le soleil. D'autres comètes ont montré des particularités semblables que Vsekhsvyatsky rapproche des rayons coronaux. Il suggère que la production des synchrones pourrait être dû à des champs électromagnétiques. Cette communication est discutée par Levin, Biermann et Wurm.

D. O. Mokhnatch lit une communication intitulée 'On the question of planning of cometary observations'. Il rappelle les travaux qu'il a publiés depuis I938 sur la distribution de la luminosité dans les têtes cométaires. Il insiste sur la nécessité de préparer un programme de photographies à travers des filtres laissant passer des bandes couvrant des 


\section{COMMISSION 15}

émissions bien définies. Il signale l'importance, pour l'Astrométrie, de la non-coïncidence du centre d'inertie et du centre photométrique de la tête.

L. Panajotov décrit les spectres de $1956 h$ qu'il a obtenus au prisme-objectif. Liller expose sa méthode d'enregistrement photoélectrique de spectres cométaires pris au prisme-objectif et ses résultats pour les comètes de 1957, en particulier en ce qui concerne la distribution d'intensité dans le continuum. La même question (spectrophotométrie du continuum) est exposée par Madame R. Herman sur la base de ses clichés de I956h.

On entend ensuite quatre exposés sur les mesures de polarisation par Richter, Vanyšek, Mirzoyan-Khatchikian et Bappu. Ceux-ci donnent lieu à une intéressante discussion.

Enfin Nazartchouk relate ses observations du mouvement des concentrations nuageuses dans la queue de la comète I956h.

En terminant cette dernière séance, le Président exprime ses remerciements à tous ses collègues de la Commission I5 pour l'aide précieuse qu'ils lui ont apportée au cours de ses six années de présidence.

\section{Réunion mixte des Commissions 7, I5, I6, 20 et 22 tenue le I8 août I958}

Cette conférence organisée par le Professeur Vsekhsvyatsky a remporté un grand succès. Les communications suivantes ont été exposées:

S. K. Vsekhsvyatsky: Introduction;

J. H. Oort and Mrs Van Houten-Groeneveld: On the distribution of reciprocal major axes of long-period comets (interventions de Lyttleton, Bronstein et Vsekhsvyatsky);

G. P. Kuiper: On the origin of comets (interventions de Levin et Urey);

R. A. Lyttleton: The formation of comets from interstellar dust (interventions de Witkowski, Levin, Oort, Bondi et Swings);

F. L. Whipple: Condensation; notes on comets, meteors and planetary evolution (interventions de Witkowski et Lebedinsky);

V. G. Fesenkov: Zodiacal light as product of the desintegration of asteroids (intervention de Cherednichenko);

N. Richter: Experimental determination of the optical constants of interplanetary matter (intervention de Lebedinsky);

K. Steins: The problem of capture of comets (interventions de Whipple et Lyttleton);

S. K. Vsekhsvyatsky: Comets and the problems of the solar system;

V. I. Cherednichenko: Lifetime of periodic comets;

I. S. Astapovich: Rate of evolution of meteoric systems;

L. Kresák: On the age determination of meteor streams;

J. G. Davies: The orbits of faint meteors, and their association with comets.

La communication annoncée de A. Dauvillier (Sur l'existence de deux familles cométaires) n'a pas été entendue, étant donné l'absence de l'auteur. 